\title{
DISPOSICIÓN DE LOS AGRICULTORES A IMPLANTAR MÁRGENES MULTIFUNCIONALES EN SU EXPLOTACIÓN. EL CASO DE LOS SECANOS ANDALUCES
}

\author{
$\underline{\text { Rubén Granado-Díaz }}^{\mathrm{a}, \mathrm{b} *}$, Teresa Parra ${ }^{\mathrm{a}}$, Manuel Estévez ${ }^{\mathrm{a}}$, Trinidad Manrique ${ }^{\mathrm{a}}$ \\ ${ }^{a}$ Agencia de Gestión Agraria y Pesquera de Andalucía (AGAPA). Avda. Menéndez Pidal, s/n Campus \\ Agroalimentario Alameda del Obispo. 14004. Córdoba, España \\ ${ }^{b}$ WEARE-Water, Environmental and Agricultural Resources Economics Research Group. Universidad de \\ Córdoba. Campus Rabanales, Ctra. N-IV. 14014. Córdoba, España
}

\begin{abstract}
Resumen
La Política Agraria Común (PAC) se está orientando hacia la consecución de objetivos medioambientales, como lo muestra la inclusión de los "Regímenes en favor del clima y del medio ambiente" (ecoesquemas) en los pagos directos de la futura PAC. Dentro de las prácticas disponibles para este fin en los cultivos herbáceos de secano destacan los "Márgenes multifuncionales", entendidos como franjas de terreno con vegetación espontánea o sembrada implantados en los márgenes de las parcelas para mejorar la biodiversidad. Esta práctica está presente en una medida del Programa de Desarrollo Rural de Andalucía y se plantea su inclusión dentro de los ecoesquemas de la futura PAC. Con el objetivo de analizar la posibilidad de extenderla entre los secanos andaluces, se ha realizado un ejercicio de valoración contingente doble dicotómica a partir de una encuesta a agricultores. A partir de esta encuesta, y aplicando un modelo econométrico, se ha estimado la disposición de los agricultores a aceptar esta práctica. Los resultados muestran que su generalización requeriría importes superiores a los obtenidos mediante pérdida de ingresos y costes adicionales utilizados hasta ahora en las medidas de agroambiente y clima, lo que también tiene implicaciones para el diseño de los ecoesquemas.
\end{abstract}

Palabras clave: Política Agraria Común, regímenes en favor del clima y del medio ambiente, medidas de agroambiente y clima, valoración contingente doble dicotómica, disposición a aceptar.

\section{Introducción}

La Política Agraria Común (PAC) se ha ido orientando cada vez más hacia la consecución de objetivos de carácter medioambiental. Fruto de esta orientación, las medidas de agroambiente y clima han tomado un mayor protagonismo dentro de los Programas de Desarrollo Rural (PDR) y se han introducido medidas de carácter medioambiental también en los pagos directos, como es el caso del actual pago por prácticas agrícolas beneficiosas para el clima y el medio ambiente (pago verde o greening) y los "Regímenes en favor del clima y del medio ambiente" (ecoesquemas) que lo sustituirán en la futura PAC.

En este contexto, es necesario disponer de una gama amplia de prácticas agrarias beneficiosas para el medio ambiente a disposición de los agricultores, de forma que todos los tipos de explotaciones puedan acceder a este tipo de pagos contribuyendo así de forma significativa a mejorar el desempeño ambiental de la agricultura.

En el caso de los cultivos herbáceos de secano, la implantación de márgenes multifuncionales puede resultar una práctica de interés. Los márgenes multifuncionales son franjas de terreno situadas en los márgenes de las parcelas agrícolas en las que está presente vegetación espontánea o sembrada de diferentes especies. La presencia de esta vegetación presenta grandes beneficios ambientales, especialmente en cuanto al mantenimiento de la biodiversidad de las tierras agrarias (polinizadores, aves, reptiles, pequeños mamíferos...) [Marshall y Moonen (2002)], además de presentar otros beneficios agronómicos como la protección del suelo.

Esta práctica está ya incluida dentro de la medida 10.1.4 (Sistemas sostenibles de cultivos herbáceos de secano) del PDR de Andalucía 2014 - 2020, así como en la propuesta de aplicación de los nuevos ecoesquemas por parte del Ministerio de Agricultura y Pesca [MAPA, (2021)]. No obstante, la medida del PDR andaluz ha tenido una escasa aceptación entre los beneficiarios potenciales.

En el caso de la medida del PDR, el importe de la prima se obtiene a partir de la pérdida de ingresos y los costes adicionales que supone esta práctica respecto a la situación en la que esta no se aplicase, según la normativa comunitaria. En este sentido, la implantación de estos márgenes supone para el agricultor en primer lugar una reducción de sus ingresos derivada de la pérdida de producción en la superficie en la que 
el margen estuviese presente, al no poder compatibilizarse con la producción agraria en esa misma superficie. Por otro lado, supone además un incremento de costes asociado a la implantación y mantenimiento del margen.

Unido a esto, el agricultor puede percibir la existencia de otros costes de difícil evaluación, como los asociados a un mayor riesgo de plagas y enfermedades en el cultivo adyacente, o al riesgo de "descontrol" de la vegetación presente en el margen que pudiese invadir el resto de la parcela [Marshall y Moonen (2002)]. Estos costes pueden explicar en parte la escasa aceptación de esta práctica por parte de los agricultores.

Por este motivo resulta necesario analizar la disposición de los agricultores andaluces de secano a implementar los márgenes multifuncionales, lo que permitiría compararla con la estimación de pérdida de ingresos y costes adicionales y adecuar el diseño de las futuras medidas de agroambiente y clima y los ecoesquemas.

\section{Metodología}

Esta investigación se ha basado en las respuestas recopiladas de una encuesta telefónica realizada a una muestra representativa de agricultores $(\mathrm{N}=102)$ que hubiesen presentado una solicitud única de ayudas de la PAC en la campaña 2020 y que tuviesen, entre sus cultivos declarados, cultivos elegibles para la medida 10.1.4 del PDR de Andalucía en tierras de secano. El cuestionario contenía información general sobre la explotación, el conocimiento de la medida 10.1.4 y el conocimiento del agricultor respecto de los márgenes multifuncionales.

Para analizar la aceptación de la implantación de estos márgenes por los agricultores, se incluyó un ejercicio de valoración contingente doble dicotómico [Hanemann et al. (1991)]. En este ejercicio, se les explicó en primer lugar en qué consistían los márgenes multifuncionales, indicándoles que, si aceptaban implantarlos tendrían que hacerlo en un 5\% de la superficie de cultivos herbáceos y que recibirían a cambio de una compensación, además de que serían compatibles con el actual pago verde (o greening). En un primer momento se ofreció una cantidad de $850 € /$ ha de margen multifuncional. En caso de que el agricultor aceptase esa oferta, se realizaba una segunda de $550 € / \mathrm{ha}$, mientras que en el caso de que la rechazase se ofrecía una oferta de $1.300 € /$ ha.

Los niveles de pagos ofrecidos se han establecido teniendo en cuenta el importe asociado a la práctica de márgenes multifuncionales de la actual medida 10.1.4.

A partir de las respuestas de estos agricultores se ajustó un modelo de diferencias en utilidad con función de utilidad lineal de Hanemann [Hanemann y Kanninen (1999)] considerando una distribución normal, utilizando el paquete maxLik del software R [Henningsen y Toomet (2011)]. Con este modelo se estimó la DAA media y su desviación estándar, a partir de las cuales puede obtenerse la proporción de agricultores dispuestos a participar en las ayudas en función del importe de la ayuda que se concediese.

\section{Resultados}

En la Tabla 1 se muestran los resultados del modelo econométrico. Los coeficientes obtenidos son altamente significativos (al 99,9\%) y tienen el signo esperado, es decir, positivo para la ayuda y negativo para la constante. A partir de estos valores, se obtiene la DAA media de los agricultores por implantar los márgenes multifuncionales (Tabla 2), la cual asciende a 669,74 €/ha de margen, así como su desviación típica (388,39 $€ /$ ha de margen). Teniendo en cuenta que la medida 10.1 .4 requiere implantar el margen en un $5 \%$ de la superficie de cultivos herbáceos (10\% para zonas RAMSAR), la DAA media para esta medida sería de $33,49 € /$ ha de cultivos herbáceos de secano (66,97 en zonas RAMSAR), mientras que la ayuda prevista en esta medida por la implantación de márgenes multifuncionales es de $26,27 € /$ ha de cultivos herbáceos de secano (52,54 en zonas RAMSAR). Se observa, por tanto, que la DAA media es superior a la ayuda calculada a partir de la pérdida de ingresos y costes adicionales previstos en la normativa comunitaria, lo cual dificulta la adopción de esta práctica por parte de los agricultores de herbáceos de secano andaluces. 
Tabla 1. Resultados del modelo econométrico.

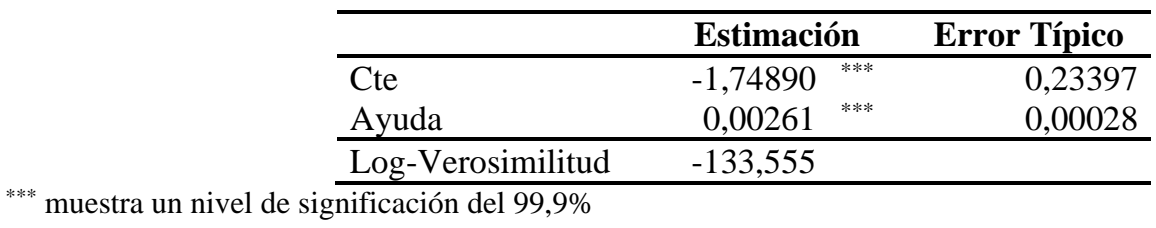

Tabla 2. DAA media y desviación típica.

\begin{tabular}{lcc}
\hline & Media (€/ha) & Intervalo Conf. 95\% \\
\hline DAA Media & $669,74^{* * * *}$ & $(598,54-737,78)$ \\
Desviación Típica & $388,39^{* * *}$ & $(326,61-465,84)$ \\
\hline
\end{tabular}

*** muestra un nivel de significación del 99,9\%. Estimaciones obtenidas siguiendo el método propuesto por Krinsky y Robb (1986) (10.000 extracciones).

Por otro lado, el modelo permite obtener una distribución probabilística con la cual estimar el grado de aceptación de la práctica en función de la ayuda ofrecida. Así, para el importe de ayuda ofrecido actualmente en la medida 10.1.4, la probabilidad de aceptación sería del 35,5\%. A este hecho hay que añadir que en la medida del PDR andaluz se incluyen otros requisitos como la obligatoriedad de realizar producción integrada y de utilizar una mezcla de semillas con especies concretas, aspectos que no se han incluido en este ejercicio de valoración contingente, por lo que es de esperar que la DAA real de los agricultores por esta medida concreta sea mayor a la estimada y, por tanto, la probabilidad de aceptación inferior.

\section{Conclusiones}

La generalización de la implantación de márgenes multifuncionales por parte de los agricultores de secano de Andalucía requeriría importes de ayuda que superan la pérdida de ingresos y los costes adicionales calculados, lo cual explicaría, al menos en parte, la escasa aceptación de la medida actual dentro del PDR que incluye esta práctica. Este hecho pone asimismo de manifiesto que el agricultor tiene en cuenta otros factores difíciles de valorar a la hora de tomar sus decisiones respecto de la participación de las medidas de agroambiente y clima.

La aplicación de técnicas de valoración como la valoración contingente resultan de interés para las administraciones públicas, ya que permite conocer no solo los valores medios sino también una distribución probabilística de la aceptación de este tipo de medidas, pudiendo estimar el porcentaje de agricultores que podrían acogerse a ellas y, con ello, hacer una estimación más precisa del presupuesto necesario. Por tanto, se hace necesario revisar la normativa comunitaria para permitir incluir estas otras metodologías de cálculo dentro de las medidas de los PDR.

Los resultados son especialmente relevantes en cuanto al diseño de los futuros ecoesquemas, ya que, si el cálculo de esta ayuda se basase exclusivamente en la pérdida de ingresos y los costes adicionales, su importe podría no ser suficiente para incentivar su adopción por gran parte de los beneficiarios potenciales, no contribuyendo a alcanzar a los objetivos medioambientales de la futura PAC. Como alternativa debería optarse por un pago incentivador, tal y como se prevé en las propuestas legislativas de la futura PAC, que permita elevar el importe de ayuda a un nivel suficiente para que sea atractivo para una mayoría de agricultores.

\section{Bibliografía}

Hanemann, M., Loomis, J. y Kanninen, B. (1991). "Statistical efficiency of double-bounded dichotomous choice contingent valuation”. American Journal of Agricultural Economics, 73(4): 1255-1263.

Hanemann, W.M. y Kanninen, B. (1999). "The Statistical Analysis of Discrete-Response CV Data". En Bateman, I.J. \& Willis, K.G. (Eds.): Valuing Environmental Preferences - Theory and Practice of the Contingent Valuation in the US, EU and Developing Countries. Oxford University Press, Oxford: 302-441.

Henningsen, A. y Toomet, O. (2011). "maxLik: A package for maximum likelihood estimation in R". Computational Statistics, 26(3): 443-458. 
Krinsky, I. y Robb, A.L. (1986). "On approximating the statistical properties of elasticities". Review of Economics and Statistics, 68(4): 715-719.

Marshall, E.J.P. y Moonen, A.C. (2002). "Field margins in northern Europe: their functions and interactions with agriculture”. Agriculture, Ecosystems \& Environment, 89(1): 5-21.

Ministerio de Agricultura Pesca y Alimentación (MAPA). (2021). ECOESQUEMA 9: PRÁCTICAS PARA LA MEJORA DE LA BIODIVERSIDAD. Documento PE GTAN FEAGA, 29.01.2021. Ministerio de Agricultura, Pesca y Alimentación, Madrid. 